\title{
WHEAT AREA MAPPING AND PHENOLOGY DETECTION USING SYNTHETIC APERTURE RADAR AND MULTI-SPECTRAL REMOTE SENSING OBSERVATIONS
}

\author{
J. D. Mohite ${ }^{1, *}$, S. A. Sawant ${ }^{1}$, S. Rana ${ }^{1}$, S. Pappula ${ }^{1}$ \\ ${ }^{1}$ TCS Research and Innovation, Tata Consultancy Services, Thane, Maharashtra, India - (jayant.mohite, suryakant.sawant, \\ sheesham.rana, srinivasu.p)@tcs.com
}

Commission III, WG III/10

KEY WORDS: Wheat area mapping, Sentinel-1, Sentinel-2, Random Forest, Classification, Google Earth Engine

\begin{abstract}
:
In season crop area mapping is of significant importance for multiple reasons such as monitoring if crop health and residue burning areas, etc. Wheat is one of the important cereal crop cultivated all across the India, with Punjab-Haryana being the prime contributors to the total production. In this study we propose a method for early season Wheat area mapping using the combined use of temporal Sentinel-1 and 2 observations. Further, we propose a method to estimate the crop phenology parameter viz. sowing date using the early time series of Normalized Difference Vegetation Index (NDVI). Few districts from Haryana and Punjab have been selected. The Wheat sowing starts in month of Oct.-Nov. Considering the sowing window, images available during Oct.-Dec. 2017 have been chosen for early season Wheat area mapping. The field data for Wheat, other crops, forest, water and settlements classes is gathered using human participatory sensing and Google Earth Engine (GEE) platform and used for data analysis. We have assessed the performance of random forest classifier using 1. NDVI derived from Sentinel-2, 2. VV and VH backscatter obtained from Sentinel-1 and 3. Both NDVI and VV-VH backscatter. Results show the maximum classification accuracy of $88.31 \%$ when using combination of NDVI, VV and VH. However, accuracy drops to $87.19 \%$ and $79.16 \%$ while using NDVI and VV-VH respectively. Further, to estimate the sowing date we have considered the NDVI time-series during Oct.-Dec. for Wheat pixels. A method based on NDVI compositing is used with gradual increase of 0.1-0.15 at every 12 days for subsequent two images. We have found a good agreement between the estimated sowing dates and actual sowing dates.
\end{abstract}

\section{INTRODUCTION}

Wheat is one of the important cereal crops cultivated in India with key contribution in terms of area and production. Globally India ranks second in wheat production after China. In India, Wheat was cultivated on 314.65 lakh hectares during 2014-15 which reduced to 304.18 and 305.97 lakh hectares during 201516 and 2016-17 respectively. However, the production has increased from 86.53 million tonnes (MT) in 2014-15 to 92.29 MT in 2015-16 and 98.38 MT during 2016-17 (Department of Agriculture, 2017). Major wheat growing states in India are Uttar Pradesh, Punjab, Haryana, Rajasthan, Madhya Pradesh, Gujarat and Bihar.

Being a major crop, there is a need to understand the spatiotemporal distribution in a timely and accurate manner. The information on spatial distribution of Wheat cropping areas is useful for stakeholders (e.g. cultivators, fertilizer/pesticide manufacturers and agriculture extension agencies) to effectively plan supply of inputs, market activities. The information on the temporal pattern in the sowing window would be helpful to the stakeholders to track the probable crop residue burning activities and plan the remedial actions to avoid/minimize the burning. In addition to this, spatio-temporal Wheat crop area maps are useful in getting the estimates on regional water demand, input to crop health and crop yield models.

Remote Sensing has been used for crop area mapping since long time that involves use of both optical as well SAR sensors on satellite platform. With advent of space technology, there are multiple satellite (optical/SAR) have been launched by many space agencies like Indian Space Research Organization (ISRO), National Aeronautics Space Administration (NASA), European Space Agency (ESA), etc. Wheat area mapping at various scales (field, regional, national) has been carried out in the past using number of approaches which involves use of single date or time series of optical data (Belgiu and Csillik, 2018; Jin et al., 2016; Nasrallah et al., 2018). Belgiua et al., (2018) evaluated the use of Time Weighted Dynamic Time Warping (TWDTW) algorithm on both pixel as well as object based classifications for mapping various crop types. The study showed that for all study areas the object based TWDTW method outperforms the pixel based TWDTW. The Support Vector Machine based model was developed by Jin et al., (2016) by using the maximum NDVI and time integrated NDVI derived from Chinese $\mathrm{HJ}-1 \mathrm{~A} / \mathrm{B}$ for mapping of rain-fed and irrigated Wheat. Nasrallah et al., (2018) proposed an innovative approach named Simple and Effective Wheat Mapping Approach (SEWMA), to map the winter Wheat areas using NDVI values of four-month period that coincides with several phenological stages of the Wheat. Further, various researchers have attempted to map the crop phenological stages using satellite based time series data (Jain et al., 2016; Zheng et al., 2016; Liu et al., 2018). Jain et al., (2016) used micro-satellite SkySat's high spatial $(2 \mathrm{~m})$ and temporal (bi-weekly) resolution observations to map the sowing dates in India. The study showed that sowing dates were predicted well while using the models that were parameterized using self-report sow dates collected close to the time of planting and when using imagery that spanned the entire growing season. Liu et al., (2018) presented an approach for real time monitoring of crop

Corresponding author 
phenology using Visible Infrared Imaging Radiometer Suite (VIIRS) observations and historical Moderate Resolution Imaging Spectroradiometer (MODIS) datasets. The Crop phenology detection framework proposed by Zheng et al., (2016) used synthetic NDVI dataset derived from fusion of Systeme Probatoire d'Observation de la Tarre5 (SPOT5) and MODIS images. We have observed that, most of the studies have either used full season time series or single date images. In addition to this most work is focused on using optical data for Wheat area mapping.

In this study an attempt has been made to combine both optical (Sentinel-2) and SAR (Sentinel-1) data for Wheat area mapping. The early season time series of RS observations during the sowing window of the crop helped to get the crop area estimates and spatial distribution of the sowing window of the crop. Further, these insights were used to train crop health/yield models. The key objective of this study is early season Wheat area mapping and phenology (start of the season) detection using multi-temporal Sentinel-2 and Sentinel-1 data. Sentinel-1A and 1B are the C-band (centre frequency: 5.405 $\mathrm{GHz}$ ) radar sensors with a 12-day revisit time and spatial resolution of $5 \mathrm{~m}$ and $20 \mathrm{~m}$ in range and azimuth directions, respectively. Sentinel-2A and $2 \mathrm{~B}$ is a satellite by ESA which acquires optical data at 10 days revisit frequency and $10 \mathrm{~m}$ to $60 \mathrm{~m}$ spatial resolution. The free availability of these high resolution satellite observations at good revisit period facilitates the Wheat area mapping. Information on study area, datasets used, and overall framework of Wheat area mapping and phenology detection has been described in Section 2. Section 3 covers details about the data analysis approach, results and classification performance using various scenarios. The summary and conclusions are described in Section 4.

\section{OPERATIONAL WHEAT AREA MAPPING FRAMEWORK}

\subsection{Study Area}

The study area is located in Punjab and Haryana states of India (Figure 1). The districts selected from the Punjab are Bhatinda, Fatehgarh Sahib, Ludhiana, Mansa, Moga, Patiala, Rupnagar and Sangrur. The districts such as Ambala, Sonepat, Yamunanagar, Kaithal, Karnal, Kurukshetra, Panchkula and Panipat are selected from Haryana. Wheat is one of the important cereal crops cultivated all across India, with PunjabHaryana being the prime contributor states to the total production. The Wheat-Rice cropping pattern is predominantly followed in most of the Punjab-Haryana. Other crops cultivated in this region are cotton, sugarcane, maize, barley, etc. Wheat is a Rabi crop which is sown in Oct.-Nov. soon after the harvesting of Rice.

\subsection{Datasets Used}

In this study we have used NDVI derived from Sentinel-2A and 2B and VV, VH backscatter from Sentinel-1A and 1B satellite imagery. The human participatory sensing platform RuPS mobile application (Mohite et al., 2015) and mKRISHI ${ }^{\circledR}$ has been used to collect the ground truth field observations. The district wise crop acreage statistics from department of agriculture Punjab and Haryana is used for comparative analysis.

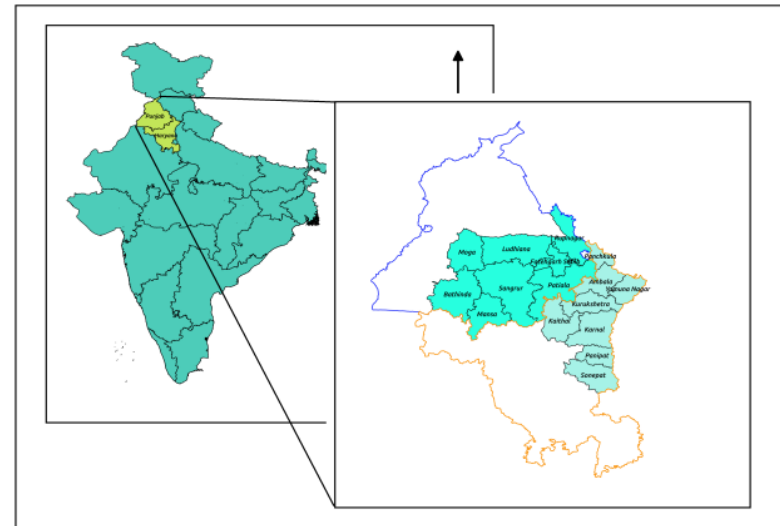

Figure 1. Study Area

2.2.1 Sentinel-2 Data and Pre-processing: Sentinel-2 is an earth observation satellite launched by ESA which captures optical imagery at high spatial resolution varying from $10 \mathrm{~m}$ to $60 \mathrm{~m}$ and has a continuous coverage of earth every 10 days with one satellite and 5 days with 2 satellites (ESA, 2017b). The data can be used for various applications such as Land Monitoring studies, including the monitoring of vegetation, soil and water cover, as well as observation of inland waterways and coastal areas (Gorelick et al., 2017). Sentinel-2 images are processed to Level-1C, which are orthorectified, map-projected images containing top-of-atmosphere reflectance data. We derived NDVI time series from the early season cloud free Sentinel-2 data available during Oct.-Dec. 2017.

2.2.2 Sentinel-1 Data and Pre-processing: ESA launched SAR satellite series Sentinel 1A and 1B. Sentinel-1 is imaging radar mission operating day and night and acquires the imagery regardless of weather. The Sentinel-1 SAR instrument operates at $5.405 \mathrm{GHz}$ (C-band corresponding to a radar wavelength of about $5.6 \mathrm{~cm}$ ), containing $\mathrm{VH}$ and VV polarizations with the revisit cycle of 12 days and a spatial resolution of $5 \mathrm{~m}$ by $20 \mathrm{~m}$ in the range and azimuth directions, respectively (ESA, 2017a). We have accessed the Sentinel-1 data on Google Earth Engine platform (Gorelick et al., 2017). The GEE has a collection of S1 Ground Range Detected (GRD) scenes, processed using the Sentinel-1 Toolbox to generate a calibrated, ortho-corrected product. Each scene is pre-processed with Sentinel-1 toolbox using the thermal noise removal, radiometric calibration and terrain correction. The final terrain corrected values are transformed to decibels $(\mathrm{dB})$ via log scaling and quantized to 16 bits. The data in VV and VH backscatter was used for early season Wheat area mapping hence images available during Oct.Dec. 2017 were used for the analysis.

2.2.3 Digital Elevation Model: The GEE has a collection of the Advanced Space-borne Thermal Emission and Reflection Radiometer (ASTER) Global Digital Elevation Model (GDEM). This was developed jointly by the U.S. National Aeronautics and Space Administration (NASA) and Ministry of Economy, Trade, and Industry (METI) Japan. The DEM has a spatial resolution of $30 \mathrm{~m}$ which was resampled to $10 \mathrm{~m}$ to match the Sentinel-1 and 2 resolution. Further, DEM tiles were mosaicked and slope in percent was estimated and used as one of the features for Wheat area mapping.

2.2.4 Ground Truth data from Field Visits: The mKRISHI ${ }^{\circledR}$ has developed an android application RuPS (Mohite et al., 2015) for participatory data collection by field staff / farmers / expert, etc. Information on plot geo-coordinates, crop cultivated 
on that plot, variety, its sowing date has been collected using the application. The information on Wheat and other crops were collected by demarcating the plot GPS boundaries. Further, the GEE interface with Google Maps overlay was used to collect the polygons on other land use classes such as water-bodies, settlements, forest and shrubs, etc. The data obtained from these sources have been used for model development and validation.

\subsection{Overall Approach}

The overall framework comprised of two main stages (Figure 2 ). The first stage involves the Wheat area mapping using temporal NDVI derived from Sentinel-2 data and VV and VH backscatter obtained from Sentinel-1 satellite. As Wheat crop is mainly grown in Rabi season in Punjab and Haryana, the Rabi season starts in Oct.-Nov. and end in Mar.-Apr. of subsequent year. In addition to this slope in percent derived from ASTER DEM has been used as a feature. Random forest classifier was trained by tuning the number of trees on GEE to achieve the best classification performance. The performance of tuned model has been tested on validation dataset. Also, independent stratified random sampling method was used for validation. The second stage involves the estimation of crop phenology parameter, that is Start of the Season (Sowing dates). We have used NDVI time series derived from cloud free Sentinel-2 images available during sowing window (Oct.-Dec.). A method based on local minima and subsequent increase in the NDVI was used for estimation of the Wheat sowing window. The estimated sowing dates were then compared with the actual sowing dates collected from the field observation of sample plots.

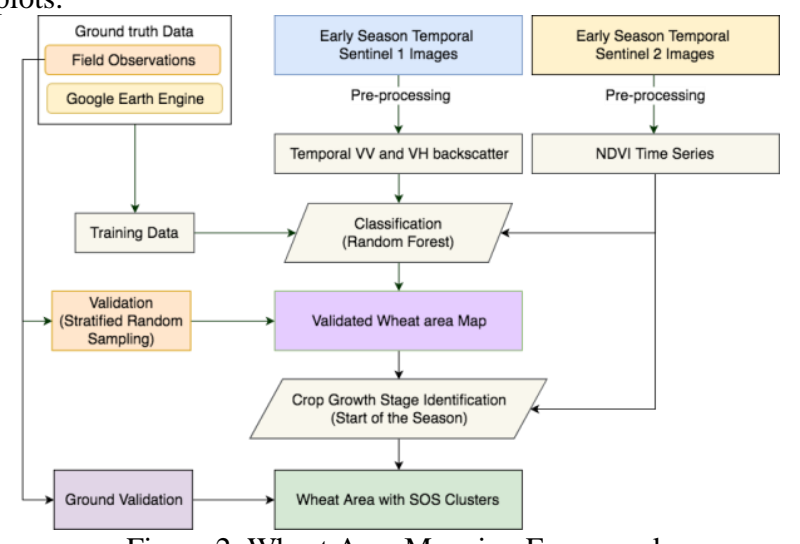

Figure 2. Wheat Area Mapping Framework

\section{RESULTS AND DISCUSSIONS}

3.1 Class Separation Across Indices: The training data of various Land Use/Land Cover (LU/LC) classes was used to extract the remote sensing time series for selected polygons. The Sentinel 1 and 2 satellite data was downloaded from GEE for the period ranging from Sept. 2017 to Apr. 2018. The LU/LC class separability plots for Sentinel-2 based NDVI and Sentinel 1 based VV and VH backscatter from Sept. 2017 to Apr. 2018 are shown in Figure 3, 4 and 5. We can observe that, monthly mean NDVI values of Wheat are clearly separated than that of other land use classes across all months except Dec. Similar separation in Wheat and other classes is observed in VV and VH polarization during all months. The distinct class separability in temporal data of NDVI, VV and VH backscatter during Oct.-Dec. 2017 was selected to obtain early season estimates of Wheat area.

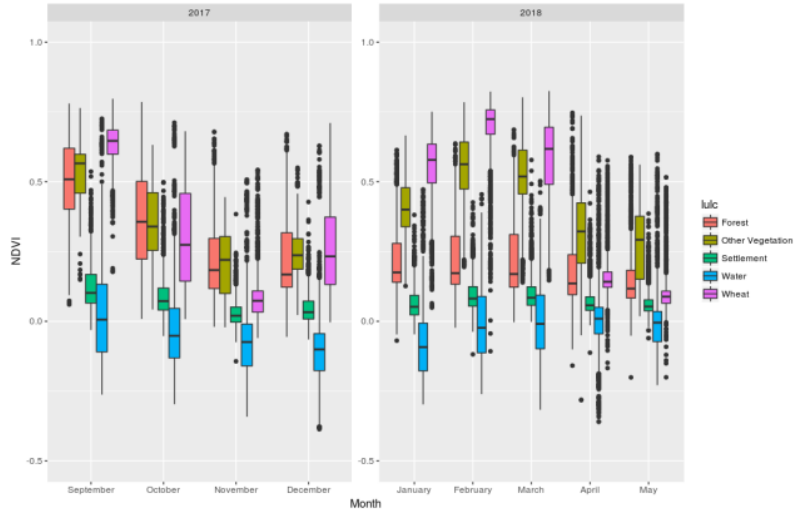

Figure 3. LU/LC Class Separability in NDVI

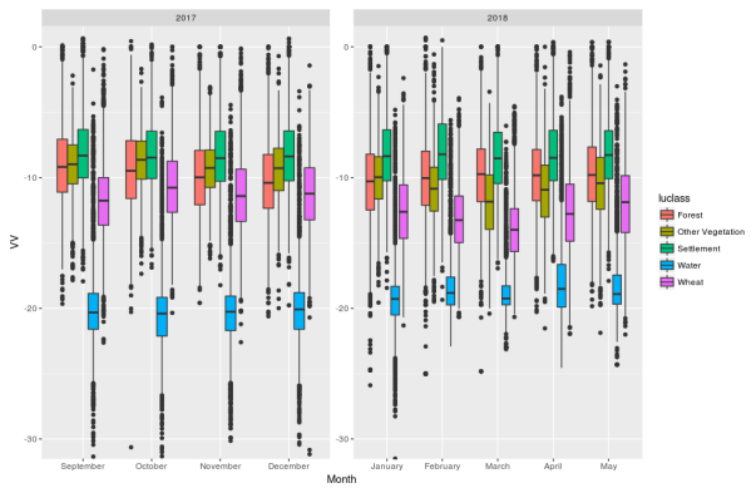

Figure 4. LU/LC Class Separability in VV Polarization

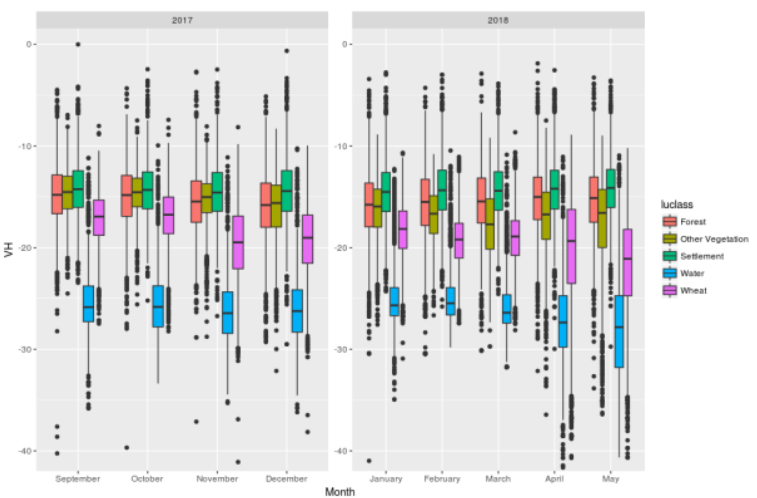

Figure 5. LU/LC Class Separability in VH Polarization

3.2 Wheat Area Mapping: Total six cloud free NDVI images and $14(7 \mathrm{VV}+7 \mathrm{VH})$ Sentinel-1 images available during Oct.Dec. 2017 along with Slope (\%) derived from ASTER were considered for Wheat area mapping and evaluating the performance of Random Forest classifier. To train classifier and assess accuracy, the ground truth field boundaries were used that comprised of total area of 1000 ha. (rice 200 ha., settlement 210 ha., forest 200 ha., water 254 ha. and other crop 145.21 ha.). The ground truth data was divided into training and validation dataset of 70 and 30 percent respectively. The feature combinations were comprised of 1 . Only NDVI during Oct. to Dec. 2017, 2. Only VV and VH during Oct. to Dec. 2017 and 3. NDVI, VV and VH during Oct. to Dec. 2017. The random forest classifier was tuned by varying the number of trees ( 5 to 60) to achieve the best performance on both training and validation data-set. The overall accuracy on validation data-set was $88.31 \%$ when using all the three features NDVI, VV and VH backscatter. However, it drops to $87.19 \%$ when using only NDVI and $79.16 \%$ when using only VV and VH backscatter. 
Improvement in the classification accuracy is achieved by combined use of Sentinel-2 derived NDVI and Sentinel-1 derived VV, VH backscatter. We have also noticed that, there was misclassification for validation samples from Sangrur district of Punjab hence there might be overestimation of Wheat in Sangrur district of Punjab. Further, the accuracy assessment performed using stratified random sampling method for Wheat vs non-Wheat classes yielded the classification accuracy of $88 \%$ (Table 1).

\begin{tabular}{|c|c|c|}
\hline Scenario & $\begin{array}{c}\text { Validation } \\
\text { Accuracy (\%) }\end{array}$ & $\begin{array}{c}\text { Stratified Random } \\
\text { Sampling Accuracy } \\
(\%)\end{array}$ \\
\hline Only NDVI & 87.19 & - \\
\hline Only VV and VH & 79.16 & - \\
\hline NDVI, VV and VH & 88.31 & 88.0 \\
\hline
\end{tabular}

Table 1. Performance of RF for multi-feature scenarios

Figure 6 and 7 shows the Wheat crop area map for Rabi 201718 generated using features from both Sentinel-2 (NDVI) and Sentinel-1 (VV and VH).

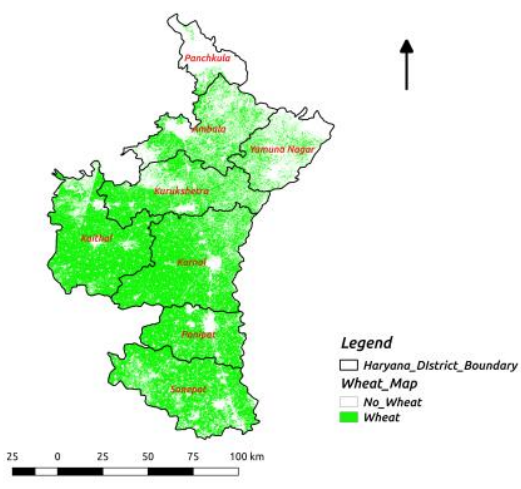

Figure 6. Wheat Classified Map for Haryana

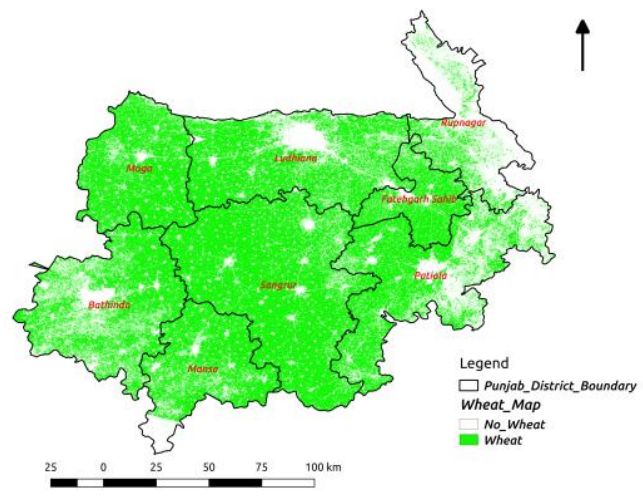

Figure 7. Wheat Classified Map for Punjab

3.3 Wheat Crop Phenology Detection (Start of the Season): Cloud free Sentinel-2 images available between Oct. to Dec. 2017 were used for estimation of Start of the Season (SOS). The pixel-wise NDVI time series was accessed on GEE to obtain the local minima during crop sowing window. Further, the positive slope between date of local minima and subsequent dates was considered. The selected date of local NDVI minima was confirmed as the SOS by using a threshold based conditional operator set to check the rise in slope (gradual increase of 0.1 to $0.15 \%)$. The method was applied on time series of each Wheat pixel. The performance of proposed method was compared with the actual date of sowing collected from the ground truth observations. The actual and estimated sowing dates were in the good agreement. In some cases it was observed that actual sowing dates were varying from estimated sowing dates due to temporal frequency of the satellite pass i.e. 5 to 10 days. Figure 8 and 9 shows the distribution of sowing dates in the study area.

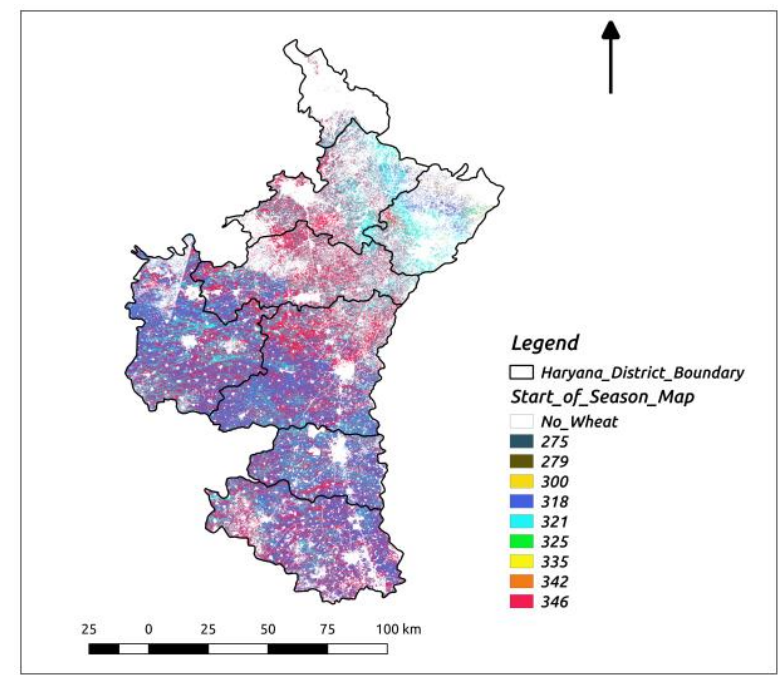

Figure 8. Wheat Start of the Season Distribution for Haryana

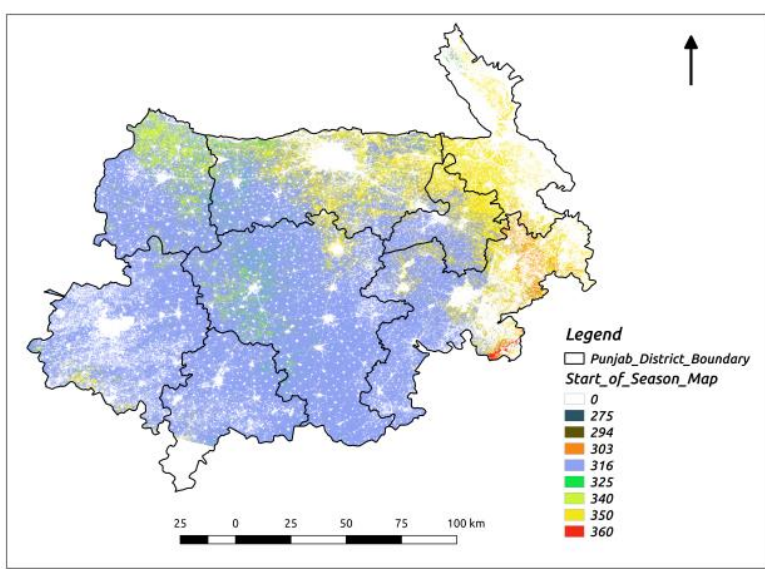

Figure 9. Wheat Start of the Season Distribution for Punjab

\section{SUMMARY AND CONCLUSIONS}

Early information on spatio-temporal extent of Wheat area and sowing pattern is very crucial to various agri-input stakeholders. The information is also helpful in getting an idea on the probable harvesting week/window which is an indicator of crop residue burning. The availability of Sentinel 1 and 2 satellite time series observations at high spatio-temporal resolution has facilitated the studies on crop area, crop health and crop phenology. In this study Wheat area has been mapped using both Sentinel-1 and 2 datasets. Also, Wheat crop phenology (start of the season) has been estimated using NDVI time series obtained from Sentinel-2 satellite observations. The study was carried out in major Wheat growing districts of Punjab and Haryana, India. Combining early season NDVI data from Sentinel-2 with VV, VH backscatter from Sentinel-1 outperformed other two approaches consisting only NDVI and only VV, VH backscatter. The best classification accuracy obtained was $88.31 \%$ on validation dataset however $88 \%$ accuracy was achieved using independent stratified random sampling. In addition to this, start of the season estimated using the proposed approach was in good agreement with the actual 
start of the season for sample fields observed during the ground validation.

\section{ACKNOWLEDGEMENTS}

Authors would like to thank research group at Research and Innovation (RnI) Lab, Tata Consultancy Services (TCS) for field observations from mobile based agro-advisory platform mKRISHI®. The Sentinel $1 \& 2$ remote sensing observations were obtained from ESA Copernicus Open Access Hub and GEE platform.

\section{REFERENCES}

Belgiu, M. and Csillik, O., 2018. Sentinel-2 cropland mapping using pixel-based and object-based time-weighted dynamic time warping analysis. Remote sensing of environment 204, pp. 509523.

Department of Agriculture, C. F. W., 2017. ANNUAL REPORT 2017-18.

http://agricoop.nic.in/sites/default/files/Krishi

ESA, 2017a. Sentinel-1.

https://sentinel.esa.int/web/sentinel/missions/sentinel-1.

ESA, 2017b. Sentinel-2.

https://sentinel.esa.int/web/sentinel/missions/sentinel-2.

Gorelick, N., Hancher, M., Dixon, M., Ilyushchenko, S., Thau, D. and Moore, R., 2017. Google earth engine: Planetary-scale geospatial analysis for everyone. Remote Sensing of Environment.
Jain, M., Srivastava, A. K., Joon, R. K., McDonald, A., Royal, K., Lisaius, M. C., Lobell, D. B. et al., 2016. Mapping smallholder wheat yields and sowing dates using micro-satellite data. Remote Sensing 8(10), pp. 860.

Jin, N., Tao, B., Ren, W., Feng, M., Sun, R., He, L., Zhuang, W. and Yu, Q., 2016. Mapping irrigated and rainfed wheat areas using multi-temporal satellite data. Remote Sensing 8(3), pp. 207.

Liu, L., Zhang, X., Yu, Y., Gao, F. and Yang, Z., 2018. Realtime monitoring of crop phenology in the midwestern united states using viirs observations. Remote Sensing.

Mohite, J., Karale, Y., Gupta, P., Kulkarni, S., Jagyasi, B. and Zape, A., 2015. RuPS: Rural participatory sensing with rewarding mechanisms for crop monitoring. In: Pervasive Computing and Communication Workshops (PerCom Workshops), 2015 IEEE International Conference on, IEEE, pp. 378-383.

Nasrallah, A., Baghdadi, N., Faour, G., Darwish, T., Belhouchette, H. and Darwich, S., 2018. A novel approach for mapping wheat areas using high resolution sentinel-2 images. Sensors.

Zheng, Y., Wu, B., Zhang, M. and Zeng, H., 2016. Crop phenology detection using high spatio-temporal resolution data fused from spot5 and modis products. Sensors 16(12), pp. 2099. 